\title{
A sufficient condition for existence of constantly nondominated trajectories in linear time-invariant systems
}

\begin{abstract}
This paper considers the multi-criteria optimal control problem in linear time-invariant systems with a single bounded input. It has been proven that, under certain assumptions, a state trajectory is non-dominated throughout the whole control process if and only if it is yielded by a control signal that belongs to a particular class of bang-bang functions. Furthermore, an explicit formula relating hyperplanes tangent to an attainable set and control switching times is presented.
\end{abstract}

Keywords: multi-criteria optimal control, attainable set, reachable set, supporting hyperplane method

\section{Introduction}

The following paper deals with a multi-criteria optimal control problem. Namely, given a dynamical system and its initial state, one aims at finding a control signal or closed-loop control law, satisfying particular constraints, which simultaneously minimizes a number of criteria. The case considered most often in the literature is an optimization problem with a vector of a finite number of criteria on the final state of the system (see, e.g., [3]). Apart from this, mulitcriteria optimization may be applied in control science to the controller tuning problem in the case of multiple performance indices [8] or multi-loop control systems [4]. However, apart from obtaining an optimal final state, one may seek to minimize the criteria throughout the whole control process. Such an approach was proposed in [11] and considered, for example, in [9]. It may be applicable to multi-criteria control problems when the final time is not known a priori. In such a case, it is reasonable to seek trajectories optimal in each time instant, which guarantees optimality at the end of the control process.

In particular, the paper considers the existence of constantly non-dominated trajectories; i.e., trajectories that are Pareto-optimal for each moment of the control process with regard to the criteria on the instantaneous state values. To the best of the author's knowledge, no

* AGH University of Science and Technology, Department of Automatic Control and Robotics, al. Mickiewicza 30, 30-059 Krakow, Poland, e-mail: mdomka@agh.edu.pl 
analytical results have been obtained in this field so far. The reasoning presented below makes use of the geometric properties of attainable sets of dynamical systems. The supporting hyperplane method originally presented in [5] is employed in order to find a relationship between the points on the boundary of an attainable set and the control signals applied to reach them. In the following paper, an expression explicitly associating control switching moments with vectors generating a hyperplane tangent to the boundary of a reachable set is formulated. This formula is then used to state a sufficient condition for the existence of Pareto-optimal trajectories.

\section{Problem statement}

Let us consider a finite-dimensional time-invariant dynamical system given by the following equations:

$$
\begin{aligned}
\dot{x}(t) & =f(x(t), u(t)) \\
x(0) & =x_{0}
\end{aligned}
$$

where $x(t) \in \mathbb{R}^{n}, u(t) \in U \subset \mathbb{R}^{m}$ and $f: \mathbb{R}^{n} \times \mathbb{R}^{m} \rightarrow \mathbb{R}^{n}$

Set $U$ corresponds to the constraints on the instantaneous control values. Furthermore, we will consider control to be admissible if it is piecewise continuous. Let $X(t)$ now denote the set of states accessible at moment $t$ via some admissible control. Finally, let us introduce a system of $k$ criteria on the instantaneous state values $J_{i}(\cdot)$ to be minimized.

$$
J_{i}: \mathbb{R}^{n} \rightarrow \mathbb{R} \text { for } i=1,2, \ldots, k
$$

Definition 1. Point $x \in X(t)$ is dominated with regard to criteria $J_{i}$ if there exists a point $\hat{x} \in X(t)$, such that, for each $i=1, \ldots, k: J_{i}(x)>J_{i}(\hat{x})$. The point is non-dominated (Pareto-optimal) if it is not dominated.

By $P(X(t), J)$ we will denote the set of non-dominated points of $X(t)$ with regard to criteria $J_{i}$.

We aim at determining a condition on the system and criteria under which there exists a trajectory $x(\cdot)$ yielded by admissible control $u(\cdot)$ that is non-dominated for each $t \geq 0$. In such a case, the following is satisfied:

$$
\forall t \geq 0 \quad u(t) \in U \wedge x(t) \in P(X(t), J) \text {, and } u \text { is piecewise continuous }
$$

where $u(t)$ and $x(t)$ satisfy (1-2).

In the paper, we will consider a linear system with a single input bounded by $<1>$.

$$
\dot{x}(t)=A x(t)+B u(t)
$$


$A \in \mathbb{R}^{n \times n}, B \in \mathbb{R}^{n}, u(t) \in[-1,1] \subset \mathbb{R}$. Without loss of generality, we may consider the initial condition for system (4) to be equal to zero. Furthermore, let us assume that $A$ has only real eigenvalues and pair $(A, B)$ is controllable.

\section{Geometric properties of attainable set}

Under the assumptions made in the previous section, the following lemma holds $[2,10]$ :

Lemma 1. For each $\tau_{1}<\tau_{2}<\ldots<\tau_{n}$, vectors $e^{A \tau_{i}} B$ are linearly independent.

Let us fix $t>0$, which will be considered through the end of the section. Attainable set $X(t)$ is compact and convex. Furthermore, it has the following property:

Lemma 2. $X(t)$ has non-empty interior.

Proof. Let $S^{n-1}$ denote a unit sphere in $\mathbb{R}^{n}$. For each $x \in S^{n-1}$, there exists control $u_{x}$ (possibly not satisfying $\left.u_{x}(\tau) \in[-1,1]\right)$ yielding $x$ in time $t$. Control $u_{x}$ satisfies the following equation [7]:

$$
u_{x}(\tau)=B^{T} e^{-A^{T} \tau}\left(\int_{0}^{t} e^{-A s} B B^{T} e^{-A^{T} s} d s\right)^{-1} e^{-A t} x
$$

Since the system is controllable, the matrix equal to the integral in (5) is nonsingular and (5) is well-defined. Let us consider function $M: S^{n-1} \ni x \mapsto \sup _{\tau}\left|u_{x}(\tau)\right| \in \mathbb{R}$. It follows from (5) that $M$ is continuous; since its domain is compact, it has a maximum $m>0$. Thus, each point of ball $B_{m}^{n}=\left\{x \in \mathbb{R}:\|x\| \leq \frac{1}{m}\right\}$ can be obtained via a control satisfying $u_{x}(\tau) \in[-1,1]$ and $B_{m}^{n} \subset X(t)$. As a consequence, $0 \in$ int $X(t)$.

Because $X(t)$ is compact and convex, it is determined by its boundary $\partial X(t) \subset X(t)$, for which the following theorem is true $[2,5]$ :

Theorem 1. Point $x_{b}$ belongs to $\partial X(t)$ if and only if it can be reached by control $u_{b}$ satisfying:

(i) $\left|u_{b}(\tau)\right| \equiv 1$,

(ii) $u_{b}$ has $n-1$ discontinuities at most.

Furthermore, for a given point on the boundary, the control yielding it is unique up to isolated time moments.

Proof. Let us first prove the only if part. Let $x_{b}$ be a fixed point from $\partial X(t)$. We will show that control $u_{b}$ yielding $x_{b}$ satisfies $(i)$ and $(i i)$. Since $X(t)$ is convex, there exists a hyperplane $\pi$ supporting $X(t)$ at $x_{b}$. Thus, $x_{b}$ maximizes the functional:

$$
Q(x(t))=p^{T} x(t)
$$

where $p \in \mathbb{R}^{n}$ is a non-zero vector orthogonal to $\pi$. 
By Pontryagin's maximum principle [1], $u_{b}$ satisfies:

$$
\begin{aligned}
u_{b}(\tau) & =\underset{u \in[-1,1]}{\arg \max }\left(p^{T} e^{A(t-\tau)} B u\right)=\operatorname{sgn}\left(p^{T} g(\tau)\right) \\
g(\tau) & =e^{A(t-\tau)} B
\end{aligned}
$$

Property $(i)$ follows directly from (7). Expression $p^{T} g(\tau)$ can be written as $p^{T} e^{A t} e^{-A \tau} B$. It is a well-known fact that each entry of matrix $e^{-A \tau}$ is a linear combination of terms $\tau^{r} e^{-\lambda_{j} \tau}$, where $\lambda_{j}$ are eigenvalues of $A$ and $r$ are integers. Multiplying $e^{-A \tau}$ by constant matrices $p^{T} e^{A t}$ and $B$ yields an expression that is a linear combination of $\tau^{r} e^{-\lambda_{j} \tau}$ as well. Hence, $p^{T} g(\tau)$ as a function of $\tau$ is a quasi-polynomial of a weight that is $n$ at most and either has $n-1$ zeros at most or is constantly equal to 0 . However, by Lemma 1, for any set of pairwise distinct $\sigma_{i}, i=1, \ldots, n$, vectors, $g\left(\sigma_{i}\right)$ are linearly independent; so, for some $i, p^{T} g\left(\sigma_{i}\right) \neq 0$. As a consequence, $p^{T} g(\tau)$ is nontrivial; thus, control $u_{b}(\tau)$ has $n-1$ discontinuities at most and satisfies $(i i)$. Moreover, $u_{b}$ is defined uniquely in every point where $p^{T} g(\tau) \neq 0$; i.e., almost everywhere.

To prove the if part, let us now consider a control $u_{b}$ satisfying $(i)$ and $(i i)$ with discontinuities at $\tau_{1}<\tau_{2}<\ldots<\tau_{n-1}$. Some switching times $\tau_{i}$ may be greater than $t$ so that $u_{b}$ could have fewer than $n-1$ switchings in the interval $[0, t]$. Let $p \in \mathbb{R}^{n}$ denote a vector for which the following holds:

$$
p^{T} e^{A\left(t-\tau_{i}\right)} B=0 \quad \text { for } i=1,2, \ldots, n-1
$$

By Lemma 1, a nontrivial $p$ satisfying the above always exists. In such a case, $p^{T} e^{A(t-\tau)} B$ is a nontrivial quasi-polynomial that changes sign at each switching time. Hence,

$$
u_{b}(\tau)= \pm \operatorname{sgn}\left(p^{T} e^{A(t-\tau)} B\right)
$$

by Pontryagin's maximum principle, it yields point $x_{b}$ that maximizes (6) with $p$ or $-p$. Thus, $x_{b}$ belongs to one of two hyperplanes orthogonal to $p$ supporting $X(t)$; as a consequence, $x_{b} \in \partial X(t)$.

Remark 1. The only if part is true even in the case of noncontrollable systems. Then, the equivalent condition for control to yield a point on the boundary is to satisfy $(i)$ and to have strictly fewer than $k$ discontinuities, where $k$ is the dimension of the controllability subspace. This property can be obtained easily by decomposing the state-space into controllability and noncontrollability subspaces.

Let $\mathscr{U}^{n-1}$ now denote the space of all control functions defined on interval $[0, t]$ satisfying (i) and (ii) with metric induced from $L^{1}[0, t]$. By Theorem 1, there exists a bijective function:

$$
F: \mathscr{U}^{n-1} \rightarrow \partial X(t)
$$




$$
F(u)=\int_{0}^{t} e^{A(t-\tau)} B u(\tau) d \tau
$$

Clearly, $F(u)=x(t)$, where $x(\cdot)$ is a solution of (4) for a particular $u$. The following lemmas hold:

Lemma 3. Set $\mathscr{U}^{n-1}$ is compact.

Proof. Let us consider set $\mathscr{T}=\left\{\left(\tau_{1}, \ldots, \tau_{n-1}\right) \in \mathbb{R}^{n-1}: 0 \leq \tau_{1} \leq \tau_{2} \leq \ldots \leq \tau_{n-1} \leq t\right\}$ and function $G: \mathscr{T} \rightarrow \mathscr{U}^{n-1}$ that associates vector $\left(\tau_{1}, \ldots, \tau_{n-1}\right)$ with piecewise constant control taking values:

- 1 on intervals $\left(0, \tau_{1}\right),\left(\tau_{2}, \tau_{3}\right)$, and ...

- -1 on intervals $\left(\tau_{1}, \tau_{2}\right),\left(\tau_{3}, \tau_{4}\right)$, and ...

- \pm 1 on interval $\left(\tau_{n-1}, t\right)$ depending on the evenness of $n$.

It can be easily seen that $G(\mathscr{T})$ is a subset of $\mathscr{U}^{n-1}$ which consists of control functions having either exactly $(n-1)$ discontinuities and taking value 1 in their first interval or strictly fewer than $(n-1)$ discontinuities. Hence, $\mathscr{U}^{n-1}=G(\mathscr{T}) \cup-G(\mathscr{T})$. Set $\mathscr{T}$ is compact; thus, to prove the thesis, it suffices to show that $G$ is continuous. Indeed, in such a case, $G(\mathscr{T})$ as an image of compact set under a continuous map is compact; as a consequence, $\mathscr{U}^{n-1}$ is compact as a sum of two compact sets. Let us choose $\tau^{h_{0}}=\left(\tau_{1}, \ldots, \tau_{n-1}\right), \tau^{h_{n-1}}=\left(\tau_{1}+h_{1}, \ldots\right.$, $\left.\tau_{n-1}+h_{n-1}\right), \tau^{h_{0}}, \tau^{h_{n-1}} \in \mathscr{T}$, and let $\tau^{h_{i}}$ denote vector $\left(\tau_{1}+h_{1}, \ldots, \tau_{i}+h_{i}, \tau_{i+1}, \ldots, \tau_{n-1}\right)$. We can extend map $G$ and define it for sequences of $\tau_{i}$ that are not necessarily increasing. In such a case, we define the extension as $\bar{G}(\tau)=G(\sigma(\tau))$, where $\sigma$ is such a per mutation of $\tau_{i}$ that sequence $\sigma(\tau)$ is increasing. Then:

$$
\begin{aligned}
\left\|G\left(\tau^{h_{0}}\right)-G\left(\tau^{h_{n-1}}\right)\right\| & =\left\|\bar{G}\left(\tau^{h_{0}}\right)-\bar{G}\left(\tau^{h_{1}}\right)+\bar{G}\left(\tau^{h_{1}}\right)-\bar{G}\left(\tau^{h_{2}}\right)+\ldots+\bar{G}\left(\tau^{h_{n-2}}\right)-\bar{G}\left(\tau^{h_{n-1}}\right)\right\| \leq \\
& \leq\left\|\bar{G}\left(\tau^{h_{0}}\right)-\bar{G}\left(\tau^{h_{1}}\right)\right\|+\left\|\bar{G}\left(\tau^{h_{1}}\right)-\bar{G}\left(\tau^{h_{2}}\right)\right\|+\ldots+\left\|\bar{G}\left(\tau^{h_{n-2}}\right)-\bar{G}\left(\tau^{h_{n-1}}\right)\right\|
\end{aligned}
$$

Functions $\bar{G}\left(\tau^{h_{i}}\right)$ and $\bar{G}\left(\tau^{h_{i+1}}\right)$ take different values on the interval whose bounds are $\tau_{i+1}$ and $\tau_{i+1}+h_{i+1}$. As a consequence:

$$
\left\|\bar{G}\left(\tau^{h_{i}}\right)-\bar{G}\left(\tau^{h_{i+1}}\right)\right\| \leq 2\left|h_{i+1}\right|
$$

and finally:

$$
\left\|G\left(\tau^{h_{0}}\right)-G\left(\tau^{h_{n-1}}\right)\right\| \leq 2\left(\left|h_{1}\right|+\left|h_{2}\right|+\ldots+\left|h_{n-1}\right|\right)=2\left\|\tau^{h_{0}}-\tau^{h_{n-1}}\right\|_{1}
$$

The last equation means that $G$ is Lipschitz continuous and completes the proof.

Lemma 4. $F$ is a homeomorphism; i.e., both $F$ and $F^{-1}$ are continuous. 
Proof. Let us choose two control functions $u_{1}$ and $u_{2}$, and let $g(\cdot)$ be defined as in (8). $\|g(\cdot)\|$ is continuous in the closed and bounded interval; thus, by the Weierstrass theorem, it has a supremum and $\forall \tau \in[0, t]\|g(\tau)\| \leq M$. Hence:

$$
\begin{aligned}
\left\|F\left(u_{1}\right)-F\left(u_{2}\right)\right\| & =\left\|\int_{0}^{t} g(\tau) u_{1}(\tau) d \tau-\int_{0}^{t} g(\tau) u_{2}(\tau) d \tau\right\|=\left\|\int_{0}^{t} g(\tau)\left(u_{1}(\tau)-u_{2}(\tau)\right) d \tau\right\| \leq \\
& \leq \int_{0}^{t}\left\|g(\tau)\left(u_{1}(\tau)-u_{2}(\tau)\right)\right\| d \tau \leq \int_{0}^{t} M\left|\left(u_{1}(\tau)-u_{2}(\tau)\right)\right| d \tau=M\left\|u_{1}-u_{2}\right\|
\end{aligned}
$$

The above means that $F$ is Lipschitz continuous; thus, it must be continuous as well.

Let us now prove that $F^{-1}$ is continuous. Let us choose sequence $\left\{w_{k}\right\}$ in $\partial X(t)$ tending to some $w$. We will show that $F^{-1}\left(w_{k}\right)$ tends to $F^{-1}(w)$. For indirect proof, let us assume that the above is not true. In such a case, there exists such a $V$ - neighborhood of $F^{-1}(w)$ in $\mathscr{U}^{n-1}$ that there exists a subsequence of $F^{-1}\left(w_{k}\right)$ whose elements do not belong to $V$. Let this subsequence be denoted by $\left\{u_{k}\right\}$. Since $\mathscr{U}^{n-1}$ is compact, $\left\{u_{k}\right\}$ has a convergent subsequence namely, $\left\{v_{k}\right\}$, which tends to some $v^{*}$. By the continuity of $F,\left\{F\left(v_{k}\right)\right\}$ tends to $F\left(v^{*}\right)$, but $\left\{F\left(v_{k}\right)\right\}$ is a subsequence of $\left\{w_{k}\right\}$, which in turn tends to $w$; thus, $F\left(v^{*}\right)=w$ and $v^{*}=F^{-1}(w)$. Finally, $\left\{v_{k}\right\}$ tends to $F^{-1}(w)$, which contradicts the fact that the elements of $\left\{v_{k}\right\}$ do not belong to $V$. This completes the proof.

Since $X(t)$ is convex, compact, and has a non-empty interior, it is homeomorphic to $n$-dimensional ball $B^{n}$, and its boundary $\partial X(t)$ is homeomorphic to sphere $S^{n-1}$. Then, by Lemma $4, \mathscr{U}^{n-1}$ is homeomorphic to $S^{n-1}$. Applying the above reasoning to a system with any dimension, we can state the following lemma:

Lemma 5. For any $k \in \mathbb{N}$, set $\mathscr{U}^{k}$; i.e., the set of bang-bang controls with $k$ switchings at most a with metric induced from $L_{1}$, is homeomorphic to $k$-dimensional sphere $S^{k}$.

Let us now consider set $\mathscr{U}^{n-2}$. By Lemma 5 , it is homeomorphic to sphere $S^{n-2}$. Thus, since $F$ is a homeomorphism, $F\left(\mathscr{U}^{n-2}\right)$ must be homeomorphic to $S^{n-2}$ as well. Hence, we can introduce homeomorphism $G: \partial X(t) \rightarrow S^{n-1}$, such that $G\left(F\left(\mathscr{U}^{n-2}\right)\right)=S^{n-2} \times\{0\} \subset S^{n-1}$. It can be seen easily that $S^{n-2} \times\{0\}$ divides $S^{n-1}$ into two open disjoint hemispheres: $S^{+}$ and $S^{-}$, which consist of points whose last coordinate is respectively positive and negative. In a similar manner, since $G$ is a homeomorphism, $F\left(\mathscr{U}^{n-2}\right)$ divides $\partial X(t)$ into two open disjoint parts: $G^{-1}\left(S^{+}\right)$and $G^{-1}\left(S^{-}\right)$. Let us denote them and $F\left(\mathscr{U}^{n-2}\right)$ by $H^{+}(t), H^{-}(t)$ and $R(t)$, respectively. Furthermore, without loss of generality, we may assume that, in $H^{+}(t)$, there is at least one point that can be obtained via control with value 1 in its first interval of constancy. We will prove the following lemma:

Lemma 6. $H^{ \pm}(t)$ consists of points that can be reached by control satisfying $(i)$, having exactly $n-1$ discontinuities, and taking value \pm 1 in its first interval.

Proof. The two first conditions follow directly from the previous reasoning and the way $H^{ \pm}(t)$ was defined. Let us prove the third one; we can write $H^{+}(t)=H_{+}^{+} \cup H_{-}^{+}$, where $H_{ \pm}^{+}$is a subset 
of $H^{+}(t)$ consisting of points reachable by controls with value \pm 1 in the first interval. We need to prove that $H_{-}^{+}$is empty. Since $H^{+}(t)$ is connected (being homeomorphic to the hemisphere), it is sufficient to prove that both $H_{+}^{+}$and $H_{-}^{+}$are open. Indeed, in such a case, if $H_{-}^{+}$was nonempty, it would be possible to represent $H^{+}(t)$ as a sum of two open, non-empty, and disjoint sets, which is a contradiction.

Let us thus choose arbitrary control $u^{*}$ such that $F\left(u^{*}\right) \in H_{+}^{+}$. Clearly, $u^{*}$ has exactly $n-1$ switchings. Let $I_{1}, \ldots, I_{n}$ now denote the intervals of constancy of $u^{*}$ and $l>0$ be the length of the shortest interval. Let us choose control $\hat{u} \in \mathscr{U}^{n-1}$ satisfying $\left\|\hat{u}-u^{*}\right\|<\frac{1}{2} l$. In each interval, $I_{i}$ controls $u^{*}$ and $\hat{u}$ differ on the set whose measure is smaller than $\frac{1}{2} l$, which in turn is smaller than the length of each $I_{i}$. As a consequence, $\hat{u}$ must take value 1 in some point in each of the intervals $I_{1}, I_{3}, \ldots$ and -1 in some point in $I_{2}, I_{4}, \ldots$. Hence, $\hat{u}$ has exactly $n-1$ switchings and the same sequence of signs as $u^{*}$. Thus, there exists neighborhood $V$ of $u^{*}$ in $\mathscr{U}^{n-1}$ that consists solely of controls taking value 1 in the first interval. Let $W$ finally denote some neighborhood of $F\left(u^{*}\right)$ in $\partial X(t)$ contained in $H^{+}(t)$. Such a neighborhood exists because $H^{+}(t)$ is open. Set $F(V) \cap W$ is a neighborhood of $F\left(u^{*}\right)$ in $\partial X(t)$ and is contained in $H_{+}^{+}$. As a consequence, $H_{+}^{+}$is open. Analogous reasoning may be applied to $H_{-}^{+}$and $H^{-}(t)$. This completes the proof.

Let $u_{b} \in \mathscr{U}^{n-1}$ now be a fixed control with exactly $n-1$ switchings at $\tau_{1}<\tau_{2}<\ldots<\tau_{n-1}$ with $\tau_{1}>0$ and $\tau_{n-1}<t$. Moreover, let $u_{b}$ yield point $x_{b}$. The following theorem describes the neighborhood of $x_{b}$ :

Theorem 2. The boundary of $X(t)$ is smooth in a neighborhood of $x_{b}$, and a hyperplane tangent to $\partial X(t)$ at $x_{b}$ is generated by vectors $e^{A\left(t-\tau_{i}\right)} B$ for $i=1,2, \ldots, n-1$.

Proof. Let $\varepsilon$ be a small positive number. We can consider the following function:

$$
\eta:[-\varepsilon, \varepsilon]^{n-1} \rightarrow \partial X(t)
$$

which associates vector $h=\left(h_{1}, \ldots, h_{n-1}\right)$ with $F\left(u_{b}^{h}\right)$, where $u_{b}^{h}$ is control with switchings at $\tau_{1}+h_{1}, \ldots, \tau_{n-1}+h_{n-1}$, and with the same sequence of signs as in $u_{b}$. For $h=\left(0, \ldots, h_{i}, \ldots, 0\right)$, we can calculate the following directional derivative:

$$
\begin{aligned}
d_{u_{b}}^{i}= & \lim _{h_{i} \rightarrow 0} \frac{\eta(h)-\eta(0)}{h_{i}}=\lim _{h_{i} \rightarrow 0} \frac{ \pm 1}{h_{i}}\left(\int_{\tau_{i-1}}^{\tau_{i}+h_{i}} e^{A(t-\tau)} B d \tau-\int_{\tau_{i}+h_{i}}^{\tau_{i+1}} e^{A(t-\tau)} B d \tau\right)- \\
& -\left(\int_{\tau_{i-1}}^{\tau_{i}} e^{A(t-\tau)} B d \tau-\int_{\tau_{i}}^{\tau_{i+1}} e^{A(t-\tau)} B d \tau\right)=\lim _{h_{i} \rightarrow 0} \frac{ \pm 2}{h_{i}} \int_{\tau_{i}}^{\tau_{i}+h_{i}} e^{A(t-\tau)} B d \tau
\end{aligned}
$$

By mean value theorem, there exists $\xi_{h} \in\left[\min \left(\tau_{i}, \tau_{i}+h_{i}\right), \max \left(\tau_{i}, \tau_{i}+h_{i}\right)\right]$ such that the last integral in (16) is equal to $h_{i} e^{A\left(t-\xi_{h}\right)}$. In addition, $\xi_{h} \rightarrow \tau_{i}$ when $h_{i} \rightarrow 0$; finally:

$$
d_{u_{b}}^{i}=\lim _{h_{i} \rightarrow 0} \pm 2 e^{A\left(t-\xi_{h}\right)}= \pm 2 e^{A\left(t-\tau_{i}\right)} B
$$


Since the above derivatives exist for each $i$, function $\eta$ is smooth. In addition, by Lemma 1 , all vectors $d_{u_{b}}^{i}$ are linearly independent; hence, the Jacobi matrix of $\eta$ has a full rank and $\eta$ is, thus, a local parametrization of $\partial X(t)$ in a neighborhood of $x_{b}$ [6]. As a consequence, $\partial X(t)$ is smooth in a neighborhood of $x_{b}$, and vectors $d_{u_{b}}^{i}$ span a hyperplane $\pi_{x_{b}}$ tangent to $\partial X(t)$ at $x_{b}$.

In other words, Theorem 2 states that $\partial X(t)$ is smooth in a neighborhood of points from $H^{+}(t) \cup H^{-}(t)$. An example of $X(t)$ for $n=2$ is presented in Figure 1.

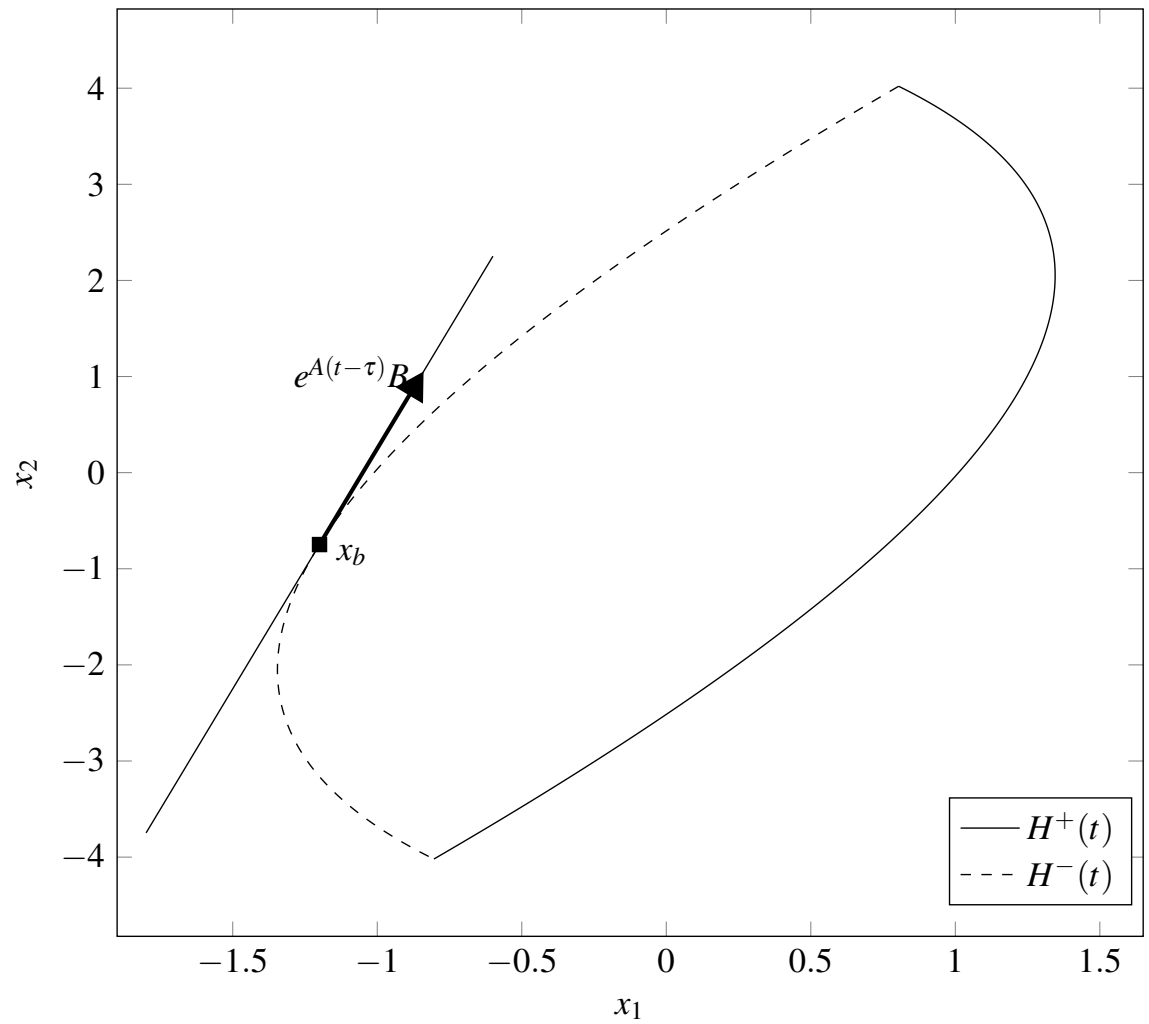

Fig. 1. Boundary of $X(t)$ with two symmetric parts drawn with solid and dashed line, respectively, and line tangent to its boundary in point $x_{b}$. Tangent line is parallel to $e^{A(t-\tau)} B$, where $\tau$ is switching time in control yielding $x_{b}$. It can be seen that $\partial X(t)$ is homeomorphic to $S^{1}$ and set of non-smooth points is homeomorphic to $S^{0}=\{-1,1\}$.

\section{Sufficient condition for existence of constantly non-dominated trajectories}

In this section, the following notation is being used: for a given set $Y$, by $z+Y$ we will denote set $\{z+y: y \in Y\}$. Similarly, $z \cdot Y$ will denote set $\{z y: y \in Y\}$. 
Let us now consider $m$ linear criteria $J_{i}$ on the instantaneous state values to be minimized. They have the following form:

$$
J_{i}(x(t))=-p_{i}^{T} x(t) \quad \text { for } i=1,2, \ldots, m
$$

for some $p_{i} \in \mathbb{R}^{n}$. Let $\theta$ denote the cone dual to the set of $p_{i}$ :

$$
\theta=\left\{p_{1}, p_{2}, \ldots, p_{m}\right\}^{*}=\left\{x \in \mathbb{R}^{n}: \forall i \quad p_{i}^{T} x>0\right\}
$$

A straightforward proof shows that the cone introduced above determines the preference structure in the considered optimization problem; that is:

$$
\forall x_{1}, x_{2} \in \mathbb{R}^{n}: \quad \forall i \quad J_{i}\left(x_{2}\right)<J_{i}\left(x_{1}\right) \Longleftrightarrow x_{2} \in x_{1}+\theta
$$

Clearly, a particular point $x$ is non-dominated if and only if $X(t) \cap(x+\theta)=\varnothing$; from this, we can gather that set $P(X(t), J)$ of all non-dominated points is included in the boundary of $X(t)$. It can be easily seen that the bang-bang control having $n-1$ discontinuities at most and taking value \pm 1 in the first interval yields state trajectory $x(\cdot)$, satisfying $\forall t \geq 0 \quad x(t) \in \overline{H^{ \pm}(t)}$. Thus, we immediately obtain a sufficient condition for the existence of non-dominated trajectories.

Theorem 3. If $\forall t \geq 0 \quad P(X(t), J)=\overline{H^{ \pm}(t)}$, constantly non-dominated state trajectories exist. Furthermore, a trajectory is constantly non-dominated if and only if it is yielded by a bangbang control having either $n-1$ discontinuities and taking value \pm 1 in the first interval or having $n-2$ discontinuities at most.

In addition, we can state the following theorem, which allows us to make use of the results obtained in Section 3:

Theorem 4. Let $\Pi$ denote the space of all hyperplanes tangent to $\partial X(t)$ for any $t$, shifted to O. Then:

$\forall t: P(X(t), J)=\overline{H^{+}(t)} \vee \forall t: P(X(t), J)=\overline{H^{-}(t)}$ if and only if, $\forall \pi \in \Pi: \pi \cap \theta=\varnothing$.

Proof. Let us first prove the if part. Let $\pi$ be an arbitrary hyperplane tangent to $\partial X(t)$ in $x_{b}$. Because of the symmetry of $X(t), \pi$ is tangent to $\partial X(t)$ in $-x_{b}$ as well. In such a case, the whole $X(t)$ is located on one side of both $x_{b}+\pi$ and $-x_{b}+\pi$, but the sides are opposite to each other. Furthermore, $x_{b}+\pi$ and $-x_{b}+\pi$ support $x_{b}+\theta$ and $-x_{b}+\theta$, respectively. Hence, either $x_{b}+\pi$ or $-x_{b}+\pi$ separates $X(t)$ from $x_{b}+\theta$ or $-x_{b}+\theta$, respectively; as a consequence, either $x_{b}$ or $-x_{b}$ are non-dominated. Because $\partial X(t)$ is piecewise smooth and $\pi$ depends continuously on $x_{b}$, all non-dominated points are located in the same smooth part; i.e., either in $\overline{H^{+}(t)}$ or $\overline{H^{-}(t)}$. The Pareto optimality of the points from $R(t)$ can be easily proven by considering a limit $x_{b} \rightarrow R(t)$. Finally, from the continuous dependence of $\overline{H^{+}(t)}$ and $\overline{H^{-}(t)}$ on $t$, we can infer that, if for some $t_{1}$, the non-dominated part is $\overline{H^{ \pm}\left(t_{1}\right)}$, then $\overline{H^{ \pm}(t)}$ is non-dominated for each $t$. 
To prove the only if part, let us assume that $P(X(t), J)=\overline{H^{+}(t)}$ and consider nondominated point $x_{b} \in H^{+}(t)$. $x_{b}+\pi$ supports $X(t)$ at $x_{b}$; since $x_{b}$ is non-dominated, $\pi$ supports $x_{b}+\theta$ as well. Thus, $\theta \cap \pi=\varnothing$.

From Theorems 2 and 4, we can immediately gather the following:

Theorem 5. If for each set of pairwise distinct and positive $\tau_{i}, \quad i=1, \ldots, n-1$ hyperplane $\operatorname{span}\left(e^{A\left(t-\tau_{i}\right)} B\right)$ is disjoint with cone $\theta$, then constantly non-dominated trajectories of the system exist, and for either $u_{0}=1$ or $u_{0}=-1$, they are yielded by a bang-bang control having either exactly $n-1$ discontinuities and taking value $u_{0}$ in the first interval or strictly fewer than $n-1$ discontinuities.

EXAMPLE. Let us consider two-dimensional system (4) with $A=\left[\begin{array}{cc}1 & -1 \\ 0 & 2\end{array}\right], B=\left[\begin{array}{l}1 \\ 1\end{array}\right]$ and criteria (18) with $p_{1}=\left[\begin{array}{ll}1 & 2\end{array}\right]^{T}, p_{2}=\left[\begin{array}{ll}1 & -3\end{array}\right]^{T}$. By Theorem 4, constantly non-dominated trajectories of the system exist if, for each $t \geq 0$, line $e^{A t} B \cdot \mathbb{R}$ is disjoint with $\theta$. Cone (19) is given by the following inequalities:

$$
\begin{aligned}
& 2 x_{2}>-x_{1} \\
& 3 x_{2}<x_{1}
\end{aligned}
$$

while the lines tangent to the attainable set are spanned by $v(t)=e^{A t} B=\left[\begin{array}{c}2 e^{t}-e^{2 t} \\ e^{2 t}\end{array}\right], t \geq 0$. It can be easily verified that inequality (21) is never satisfied by the points that belong to $v(t) \cdot \mathbb{R}$. Hence, $\forall t \quad P(X(t), J)=\overline{H^{ \pm}(t)}$ and non-dominated trajectories exist. In order to determine the value non-dominated control takes in its first interval, we can explicitly solve an optimal control problem with one criterion $J^{\prime}\left(x\left(t_{1}\right)\right)=-x_{1}\left(t_{1}\right)$ for a fixed $t_{1}$. An explicit calculation of the control yielding optimal state $x^{*}$ with regard to $J^{\prime}$ indicates that it has a switching and takes value -1 in its first interval of constancy; thus, $x^{*} \in H^{-}\left(t_{1}\right)$. On the other hand, since $x^{*}$ minimizes the convex combination of criteria $J_{1}$ and $J_{2}$, it is non-dominated at $t_{1}$ with regard to $J$ and $x^{*} \in P\left(X\left(t_{1}\right), J\right)$. Finally, $\forall t P(X(t), J)=\overline{H^{-}(t)}$, and non-dominated trajectories are yielded by either constant control functions or those having exactly one discontinuity and taking value -1 in their first interval.

\section{Conclusion}

In this paper, a multi-criteria optimization control problem for linear time-invariant systems with linear criteria was considered. An original contribution of the paper includes stating and proving a sufficient condition for the existence of constantly non-dominated trajectories, a description of hyperplanes tangent to the attainable set in function of control switching times and a proof of the topological equivalence of $(n-1)$-dimensional sphere and a space of bang-bang control functions with $(n-1)$ switchings at most. 


\section{References}

[1] Boltyanski V., Matematyczne metody sterowania optymalnego, Wydawnictwa Naukowo-Techniczne, Warszawa 1971.

[2] Boltyanski V., Gorelikova S., Optimal synthesis for nonoscillatory controlled objects, Journal of Applied Analysis 3(1), 1997, pp. 1-21.

[3] Coverstone-Carroll V., Hartmann J.W., Mason W.J., Optimal multi-objective low-thrust spacecraft trajectories, Computer Methods in Applied Mechanics and Engineering, 186(2-4), 2000, pp. 387-402.

[4] Gambier A., Wellenreuther A., Badreddin E., A New Approach to Design Multi-loop Control Systems with Multiple Controllers, in: Proceedings of the 45th IEEE Conference on Decision and Control, San Diego, 2006, pp. 1828-1833.

[5] Graettinger T.J., Krogh B.H., Hyperplane method for reachable state estimation for linear time-invariant systems, Journal of Optimization Theory and Applications, 69(3), 1991, pp. 555-588.

[6] Hairer E., Lubich C., Wanner G., Geometric Numerical Integration. Structure-Preserving Algorithms for Ordinary Differential Equations, Springer Series in Computational Mathematics, 31, Springer, Berlin 2006.

[7] Mitkowski W., Stabilizacja systemów dynamicznych, Wydawnictwa Naukowo-Techniczne, Warszawa 1991.

[8] Pedersen G.K.M., Yang Z., Multi-objective PID-controller tuning for a magnetic levitation system using NSGA-II, in: Proceedings of the Genetic and Evolutionary Computation Conference, Seattle, 2006, pp. 1737-1744.

[9] Skulimowski A.M.J., Solving Multicriteria Optimal Control Problems with Reference Multifunctions, in: 18th International Conference on Methods \& Models in Automation \& Robotics (MMAR), Międzyzdroje, 2013, pp. 115-120.

[10] Sontag E.D., Mathematical Control Theory. Deterministic Finite Dimensional Systems, Texts in Applied Mathematics, 6, Springer, New York 1998.

[11] Wierzbicki A.P., Multiobjective Trajectory Optimization and Model Semiregularization, IFAC Proceedings Volumes, 14(2), 1981, pp. 1485-1494.

\section{Warunek wystarczający istnienia stale niezdominowanej trajektorii w liniowym stacjonarnym systemie dynamicznym}

Streszczenie: $\mathrm{W}$ artykule rozważono problem wielokryterialnego sterowania optymalnego w liniowym stacjonarnym systemie dynamicznym z jednym wejściem przyjmującym wartości z ograniczonego przedziału. W szczególności udowodniono, że przy spełnieniu określonych założeń trajektoria stanu jest niezdominowana w trakcie całego procesu sterowania wtedy i tylko wtedy, gdy jest generowana przez sterowanie należące do pewnej klasy sygnałów typu bang-bang. Ponadto został przedstawiony wzór pozwalający wyrazić hiperpłaszczyznę styczną do zbioru osiągalnego w funkcji czasów przełączeń sygnału sterującego.

Słowa kluczowe: wielokryterialne sterowania optymalne, zbiór osiagalny, metoda hiperpłaszczyzn podpierajacycha 